\title{
The Stability of Solutions of Sitnikov Restricted Problem of three Bodies When the Primaries are Triaxial Rigid Bodies
}

\author{
R.R. Thapa \\ Dept. of Mathematics, P.G.Campus, Biratnagar, Morang, Nepal. \\ Email: thaparajuram@yahoo.com
}

\begin{abstract}
The paper deals with the stability of the solutions of Sitnikov's restricted problem of three bodies if the primaries are triaxial rigid bodies. The infinitesimal mass is moving in space and is being influenced by motion of two primaries $\left(\mathrm{m}_{1}>\mathrm{m}_{2}\right)$. They move in circular orbits without rotation around their centre of mass. Both primaries are considered as axis symmetric bodies with one of the axes as axis of symmetry whose equatorial plane coincides with motion of the plane. The synodic system of co-ordinates initially coincides with inertial system of co-ordinates. It is also supposed that initially the principal axis of the body $\mathrm{m}_{1}$ is parallel to synodic axis and are of the axes of symmetry is perpendicular to plane of motion.
\end{abstract}

Keywords-Axis symmetric bodies; equatorial plane; infinitesimal body; Libration points; synodic axes.

\section{INTRODUCTION}

The Sitnikov problem is a special case of restricted three body problem .It refers to the motion of the test particle along an axis perpendicular to the plane of motion of two equal primaries that move on elliptic orbits. The axis passes through the center of mass of the system.

The restricted problem of three bodies if primaries are oblate spheroids where equatorial plane coincides with plane of motion and their stability has been studied by Vidyakin (1974). Subba Rao and Sharma (1975) have studied the stability of libration points. EI-Shaboury (1991) also studied the stability of libration points. Khanna and Bhatnagar (2001) have studied the stability of solutions of Sitnikov restricted problem of the smaller primary is a triaxial rigid body.

The stability of motion in the Sitnikov problem have studied by Soulis (2007) and found that as mass of infinitesimal body increases, the domain of allowed motion grows significantly. This paper tries to establish the solutions of Sitnikov restricted problem of these bodies when both the primaries are triaxial rigid body.

\section{Solution by Lindstedt-Poincare Method}

Now following Thapa and Hassan (2013)

$$
\begin{aligned}
z= & c \cos \tau+\frac{c^{3}}{32} \cos \tau-\cos 3 \tau\left(\frac{\varepsilon}{\eta_{0}^{2}}\right) \\
& +\frac{c^{5}}{1024} 23 \cos \tau-24 \cos 3 \tau+\cos 5 \tau\left(\frac{\varepsilon}{\eta_{0}^{2}}\right)^{2}+\frac{547 c^{7}}{32768} \cos \tau\left(\frac{\varepsilon}{\eta_{0}^{2}}\right)^{3} \\
& +\frac{c^{7}}{2048}\left(-\frac{297}{8} \cos 3 \tau+3 \cos 5 \tau-\frac{\cos 7 \tau}{16}\right)\left(\frac{\varepsilon}{\eta_{0}^{2}}\right)^{3}+\ldots
\end{aligned}
$$

$$
\text { But } \begin{aligned}
\frac{\varepsilon}{\eta_{0}^{2}} & =\frac{\frac{48}{a^{7}} a^{2}+5 \alpha+10\left(\sigma_{1}+\sigma_{1}^{\prime}\right)}{\frac{8}{a^{5}} a^{2}+3 \alpha} \\
& =\frac{48}{a^{7}} \times \frac{a^{5}}{8} \frac{a^{2}+5 \alpha+10\left(\sigma_{1}+\sigma_{1}^{\prime}\right)}{a^{2}+3 \alpha} \\
& =\frac{6}{a^{2}} \frac{a^{2}+5 \alpha+10\left(\sigma_{1}+\sigma_{1}^{\prime}\right)}{a^{2}\left(1+\frac{3 \alpha}{a^{2}}\right)} \\
& =\frac{6}{a^{2}} a^{2}+5 \alpha+10\left(\sigma_{1}+\sigma_{1}^{\prime}\right)\left(1+\frac{3 \alpha}{a^{2}}\right)^{-1} \\
& =\frac{6}{a^{4}} a^{2}+5 \alpha+10\left(\sigma_{1}+\sigma_{1}^{\prime}\right)\left(1-\frac{3 \alpha}{a^{2}}+\ldots\right) \\
& =\frac{36}{\eta^{2}}\left(a^{2}+4 \alpha\right)+\frac{720}{a^{6}}\left(\sigma_{1}+\sigma_{1}^{\prime}\right) \\
& =\frac{6}{a^{4}}\left(\frac{6}{\eta^{4}}\left(a^{2}+2 \alpha\right)+10\left(\sigma_{1}+\sigma_{1}^{\prime}\right)\right]\left[\frac{36}{\eta^{6}}\left(a^{2}+4 \alpha\right)+\frac{720}{a^{6}}\left(\sigma_{1}+\sigma_{1}^{\prime}\right)\right] \\
= & \frac{6}{a^{4}}\left(a^{2}+2 \alpha\right)+10\left(\sigma_{1}+\sigma_{1}^{\prime}\right) \\
& =\frac{36}{a^{8}}\left(a^{4}+4 a^{2} \alpha+4 \alpha^{2}\right)+2 \cdot \frac{36}{a^{8}}\left(a^{2}+2 \alpha\right) \cdot 10\left(\sigma_{1}+\sigma_{1}^{\prime}\right)
\end{aligned}
$$


From (1)

$$
\begin{aligned}
z= & c \cos \tau+\frac{3 c^{3}}{16 a^{2}} \cos \tau-\cos 3 \tau+\frac{5 c^{3}}{16} \cos \tau-\cos 3 \tau\left(\sigma_{1}+\sigma_{1}^{\prime}\right) \\
& +\frac{c^{5}}{a^{4}} \frac{9}{256} 23 \cos \tau-24 \cos 3 \tau+\cos 5 \tau \\
& +\frac{c^{5}}{a^{2}} \frac{15}{128} 23 \cos \tau-24 \cos 3 \tau+\cos 5 \tau\left(\sigma_{1}+\sigma_{1}^{\prime}\right) \\
& +\frac{14769}{4096} \frac{c^{7}}{a^{6}} \cos \tau+\frac{393840}{32768} \frac{c^{7}}{a^{4}} \cos \tau\left(\sigma_{1}+\sigma_{1}^{\prime}\right) \\
& +\frac{c^{7}}{a^{6}} \frac{216}{2048}\left(-\frac{297}{8} \cos 3 \tau+3 \cos 5 \tau-\frac{1}{16} \cos 7 \tau\right) \\
& +\frac{c^{7}}{a^{4}} \cdot \frac{720}{2048}\left(-\frac{297}{8} \cos 3 \tau+3 \cos 5 \tau-\frac{1}{16} \cos 7 \tau\right)\left(\sigma_{1}+\sigma_{1}^{\prime}\right) \\
+\alpha & {\left[\frac{c^{3}}{a^{4}} \cdot \frac{3}{8}(\cos \tau-\cos 3 \tau) \frac{c^{5}}{a^{6}} \cdot \frac{144}{1024}(2 \cos \tau-24 \cos 3 \tau+\cos 5 \tau)\right.} \\
+ & \left.\frac{547 \times 216 \times 6}{32768} \frac{c^{7}}{a^{8}} \cos \tau+\frac{c^{7}}{a^{8}} \frac{216 \times 6}{2048}\left(-\frac{297}{8} \cos 3 \tau+3 \cos 5 \tau-\frac{1}{16} \cos 7 \tau\right)+\ldots\right]
\end{aligned}
$$

\section{Stability of the motion}

The general equations of motion of the infinitesimal mass under the mutual gravitational field of two axis symmetric bodies (triaxial rigid bodies) are

$$
\begin{aligned}
& \ddot{x}-2 x \dot{y}=\frac{\partial \Omega}{\partial x} \\
& \ddot{y}+2 n \dot{x}=\frac{\partial \Omega}{\partial y} \\
& \ddot{z}=\frac{\partial \Omega}{\partial z}
\end{aligned}
$$

When

$$
\Omega=\frac{1}{\left(z^{2}+\frac{a^{2}}{4}\right)^{\frac{1}{2}}}+\frac{\alpha}{4\left(z^{2}+\frac{a^{2}}{4}\right)^{\frac{3}{2}}}-\frac{3 \sigma_{1}+\sigma_{2}^{\prime} z^{2}}{4\left(z^{2}+\frac{a^{2}}{4}\right)^{\frac{5}{2}}}
$$

Where $\alpha=2 \sigma_{1}-\sigma_{2}+2 \sigma_{1}^{\prime}-\sigma_{2}^{\prime}$

Since $\Omega$ is independent of $x$ and $y$, but depending upon $z$ only

$$
\text { hence, } \begin{aligned}
\frac{\partial \Omega}{\partial x} & =0 \\
\frac{\partial \Omega}{\partial y} & =0 \\
\frac{\partial \Omega}{\partial z} & =-\frac{z}{\left(z^{2}+\frac{a^{2}}{4}\right)^{\frac{3}{2}}}-\frac{3 \alpha z}{4\left(z^{2}+\frac{a^{2}}{4}\right)^{\frac{5}{2}}}+\frac{15\left(\sigma_{1}+\sigma_{1}^{\prime}\right) z^{3}}{4\left(z^{2}+\frac{a^{2}}{4}\right)^{\frac{7}{2}}}
\end{aligned}
$$

$$
\begin{aligned}
& \text { Also } \frac{\partial^{2} \Omega}{\partial x^{2}}=0, \quad \frac{\partial^{2} \Omega}{\partial x \partial y}=0, \frac{\partial^{2} \Omega}{\partial x \partial z}=0 \\
& \frac{\partial^{2} \Omega}{\partial y^{2}}=0, \quad \frac{\partial^{2} \Omega}{\partial y \partial z}=0 \\
& \frac{\partial^{2} \Omega}{\partial z^{2}}=-\left[\frac{\left(z^{2}+\frac{a^{2}}{4}\right)^{\frac{3}{2}}-z \frac{3}{2}\left(z^{2}+\frac{a^{2}}{4}\right)^{\frac{1}{2}} 2 z}{\left(z^{2}+\frac{a^{2}}{4}\right)^{3}}\right] \\
& -\frac{3 \alpha}{4}\left[\frac{\left(z^{2}+\frac{a^{2}}{4}\right)^{\frac{5}{2}}-z \frac{5}{2}\left(z^{2}+\frac{a^{2}}{4}\right)^{\frac{3}{2}} 2 z}{\left(z^{2}+\frac{a^{2}}{4}\right)^{5}}\right] \\
& +\frac{15\left(\sigma_{1}+\sigma_{1}^{\prime}\right)}{4}\left[\frac{\left(z^{2}+\frac{a^{2}}{4}\right)^{\frac{7}{2}} 3 z^{2}-z^{3} \frac{7}{2}\left(z^{2}+\frac{a^{2}}{4}\right)^{\frac{5}{2}} 2 z}{\left(z^{2}+\frac{a^{2}}{4}\right)^{7}}\right] \\
& =-\frac{1}{\left(z^{2}+\frac{a^{2}}{4}\right)^{\frac{3}{2}}}+\frac{3 z^{2}}{\left(z^{2}+\frac{a^{2}}{4}\right)^{\frac{5}{2}}}-\frac{3 \alpha}{4\left(z^{2}+\frac{a^{2}}{4}\right)^{\frac{5}{2}}} \\
& +\frac{15 \alpha z^{2}}{4\left(z^{2}+\frac{a^{2}}{4}\right)^{\frac{7}{2}}}+\frac{45\left(\sigma_{1}+\sigma_{1}^{\prime}\right) z^{2}}{4\left(z^{2}+\frac{a^{2}}{4}\right)^{\frac{7}{2}}}-\frac{105\left(\sigma_{1}+\sigma_{1}^{\prime}\right) z^{4}}{4\left(z^{2}+\frac{a^{2}}{4}\right)^{\frac{9}{2}}} \\
& \Omega_{z z}=-\frac{1}{\left(z^{2}+\frac{a^{2}}{4}\right)^{\frac{3}{2}}}+\frac{34 z^{2}-\alpha}{4\left(z^{2}+\frac{a^{2}}{4}\right)^{\frac{5}{2}}}+\frac{15 z^{2} \alpha+3 \sigma_{1}+3 \sigma_{1}^{\prime}}{4\left(z^{2}+\frac{a^{2}}{4}\right)^{\frac{7}{2}}}-\frac{105\left(\sigma_{1}+\sigma_{1}^{\prime}\right) z^{4}}{4\left(z^{2}+\frac{a^{2}}{4}\right)^{\frac{9}{2}}}
\end{aligned}
$$

Let us write the equations as

$$
\begin{aligned}
& \ddot{x}-2 x \dot{y}=0=f(x, y, z) \\
& \ddot{y}+2 n \dot{x}=0=g(x, y, z) \\
& \ddot{z}=-\frac{z}{\left(z^{2}+\frac{a^{2}}{4}\right)^{\frac{3}{2}}}-\frac{3 \alpha z}{4\left(z^{2}+\frac{a^{2}}{4}\right)^{\frac{5}{2}}}+\frac{15\left(\sigma_{1}+\sigma_{1}^{\prime}\right) z^{3}}{4\left(z^{2}+\frac{a^{2}}{4}\right)^{\frac{7}{2}}} \\
& =h(x, y, z) .
\end{aligned}
$$

For the conditions of stable solution, the square of characteristic roots must be less then or equal to zero.

i.e. $\quad \lambda_{i}^{2} \leq 0 \quad$ for $i=1,2,3$.

But $\lambda_{1}^{2}=0 \Rightarrow \lambda_{11}=\lambda_{12}=0$.

$\lambda_{2}^{2}=-4 n^{2} \Rightarrow \lambda_{21}=2 n i, \lambda_{22}=-2 n i$.

When $\lambda_{3}^{2}=\Omega_{z z}^{0}$. 
Now,

$$
\begin{aligned}
& \Omega_{z z}^{0}=-\frac{1}{\left(z_{0}^{2}+\frac{a^{2}}{4}\right)^{\frac{3}{2}}}+\frac{34 z_{0}^{2}-\alpha}{4\left(z_{0}^{2}+\frac{a^{2}}{4}\right)^{\frac{5}{2}}}+\frac{15}{4} z_{0}^{2} \frac{\alpha+3 \sigma_{1}+3 \sigma_{1}^{\prime}}{\left(z_{0}^{2}+\frac{a^{2}}{4}\right)^{\frac{7}{2}}}-\frac{105}{4} \frac{\sigma_{1}+\sigma_{1}^{\prime} z_{0}^{4}}{\left(z_{0}^{2}+\frac{a^{2}}{4}\right)^{\frac{9}{2}}} \\
& =-\frac{1}{\left(\frac{a}{2}\right)^{3}\left(1+\frac{4 z_{0}^{2}}{a^{2}}\right)^{\frac{3}{2}}}+\frac{3}{4} \frac{4 z_{0}^{2}-\alpha}{\left(\frac{a}{2}\right)^{5}\left(1+\frac{4 z_{0}^{2}}{a^{2}}\right)^{\frac{5}{2}}}+\frac{15}{4} z_{0}^{2} \frac{\alpha+3 \sigma_{1}+3 \sigma_{1}^{\prime}}{\left(\frac{a}{2}\right)^{7}\left(1+\frac{4 z_{0}^{2}}{a^{2}}\right)^{\frac{7}{2}}} \\
& -\frac{105}{4} \frac{\left(\sigma_{1}+\sigma_{1}^{\prime} z_{0}^{4}\right)}{\left(\frac{a}{2}\right)^{9}\left(1+\frac{4 z_{0}^{2}}{a^{2}}\right)^{\frac{9}{2}}} \\
& =-\frac{8}{a^{3}}\left(1+\frac{4 z_{0}^{2}}{a^{2}}\right)^{-\frac{3}{2}}+\frac{3}{4} \times \frac{32}{a^{5}} 4 z_{o}^{2}-\alpha\left(1+\frac{4 z_{0}^{2}}{a^{2}}\right)^{-\frac{5}{2}} \\
& +\frac{15}{4} z_{0}^{2} \times \frac{128}{a^{7}} \alpha+3 \sigma_{1}+3 \sigma_{1}^{\prime}\left(1+\frac{4 z_{0}^{2}}{a^{2}}\right)^{-\frac{7}{2}} \\
& -\frac{105}{4} \times \frac{2^{9}}{a^{9}} \sigma_{1}+\sigma_{1}^{\prime} \quad z_{0}^{4}\left(1+\frac{4 z_{0}^{2}}{a^{2}}\right)^{-\frac{9}{2}} \\
& =-\frac{8}{a^{3}}+\frac{48}{a^{3}}\left(\frac{z_{0}}{a}\right)^{2}+\frac{24}{a^{3}}\left[4\left(\frac{z_{0}}{a}\right)^{2}-\frac{\alpha}{a^{2}}\right]\left[1-10\left(\frac{z_{0}}{a}\right)^{2}+\ldots\right] \\
& +\frac{15 \cdot 32}{a^{5}}\left(\frac{z_{0}}{a}\right)^{2} \alpha+3 \sigma_{1}+3 \sigma_{1}^{\prime}\left[1-14\left(\frac{z_{0}}{a}\right)^{2}+\ldots\right] \\
& -\frac{105 \times 128 \sigma_{1}+\sigma_{1}^{\prime}}{a^{5}}\left(\frac{z_{0}}{a}\right)^{4}\left[1-18\left(\frac{z_{0}}{a}\right)^{2}+\ldots\right] \\
& =-\frac{8}{a^{3}}+\frac{48}{a^{3}}\left(\frac{z_{0}}{a}\right)^{2}+\frac{24}{a^{3}}\left[4\left(\frac{z_{0}}{a}\right)^{2}-\frac{\alpha}{a^{2}}+\frac{10 \alpha}{a^{2}}\left(\frac{z_{0}}{a}\right)^{2}-\ldots\right] \\
& +\frac{480}{a^{5}}\left(\frac{z_{0}}{a}\right)^{2} \alpha+3 \sigma_{1}+3 \sigma_{1}^{\prime}\left(\frac{z_{0}}{a}\right)^{2}+\ldots \\
& =-\frac{8}{a^{3}}+\frac{48}{a^{3}}\left(\frac{z_{0}}{a}\right)^{2}+\frac{96}{a^{3}}\left(\frac{z_{o}}{a}\right)^{2}-\frac{24 \alpha}{a^{5}}+\frac{480}{a^{5}} 5 \sigma_{1}+5 \sigma_{1}^{\prime}-\sigma_{2}-\sigma_{2}^{\prime}\left(\frac{z_{0}}{a}\right)^{2}+\ldots
\end{aligned}
$$

Neglecting higher order infinitesimal above the second order

$$
\begin{aligned}
\Omega_{z z}^{0} & =-\frac{8}{a^{3}}+\frac{144}{a^{3}}\left(\frac{z_{0}}{a}\right)^{2}-\frac{24 \alpha}{a^{5}} \\
& =-\frac{1}{a^{5}}\left[8 a^{2}-144 z_{0}^{2}+24 \alpha\right] \\
& =-\frac{8}{a^{5}}\left[a^{2}-18 z_{0}^{2}+3 \alpha\right] \\
& =-\frac{8}{a^{5}}\left[a^{2}-18 z_{0}^{2}+32 \sigma_{1}-\sigma_{2}+2 \sigma_{1}^{\prime}-\sigma_{2}^{\prime}\right]
\end{aligned}
$$

Hence if $a^{2}-18 z_{0}^{2}+3 \alpha>0$ then $\Omega_{z z}^{0}<0$ and $\lambda_{3}^{2}<0$, $\lambda_{31} \& \lambda_{32}$ both are imaginary. Thus all the characteristic roots of the coefficient matrix $\mathrm{A}$ are either zero or imaginary. Hence the matrix of Sitnikov is stable.

When $a^{2}-18 z_{0}^{2}+32 \sigma_{1}-\sigma_{2}+2 \sigma_{1}^{\prime}-\sigma_{2}^{\prime}>0$

$$
\begin{aligned}
& \Rightarrow z_{0}^{2}<\frac{a^{2}+32 \sigma_{1}-\sigma_{2}+2 \sigma_{1}^{\prime}-\sigma_{2}^{\prime}}{18} \\
& \Rightarrow-\sqrt{\frac{a^{2}+32 \sigma_{1}-\sigma_{2}+2 \sigma_{1}^{\prime}-\sigma_{2}^{\prime}}{18}}<z_{0}<\sqrt{\frac{a^{2}+32 \sigma_{1}-\sigma_{2}+2 \sigma_{1}^{\prime}-\sigma_{2}^{\prime}}{18}}
\end{aligned}
$$

Thus the infinitesimal mass will perform oscillatory motion from $-\sqrt{\frac{a^{2}+3 \alpha}{18}}$ to $\sqrt{\frac{a^{2}+3 \alpha}{18}}$ about the centre of mass 0 .

\section{CONCLUSION}

If the primaries are triaxial rigid bodies then the motion of the third body depends on parameter $\tau$, distance ' $a$ ' between the primaries, constant $\mathrm{c}$ and $\alpha=2 \sigma_{1}-\sigma_{2}+2 \sigma_{1}^{\prime}-\sigma_{2}^{\prime}$. In this case, the infinitesimal mass $\mathrm{m}_{3}$ will perform oscillatory motion in between $-\sqrt{\frac{a^{2}+3 \alpha}{18}}$ to $\sqrt{\frac{a^{2}+3 \alpha}{18}}$, so that the condition of stability are satisfied by the solution of the Sitnikov motion.

\section{ACKNOWLEDGEMENTS}

The author is grateful to respected guide Dr. M.R. Hassan, Dept. of Mathematics, S.M. College, Bhagalpur, T.M.B. University, Bhagalpur, 812007, India, for his inspiration to complete this work.

\section{REFERENCES}

EI-Shaboury, S.M. 1991. Equilibrium solutions of the restricted problem of $2+2$ axisymmetric rigid bodies. Celest. Mech. Dyn. Astron. 50: 199-208.

Khanna, M. and Bhatnagar, K.B. 2001. Existence of Libration points in the restricted three body problem when the primaries are triaxial rigid bodies. Indian J. pure appl. Math. 32: 125-141.

Soulis, P.S. 2007. Stability of motion in the Sitnikov problem. Celest. Mech. Dyn. Astron. 99: 129-148.

Subba Rao, P.V. and Sharma, R.K. 1975. A note on stability of the triangular points of equilibrium in the restricted three body problem. Astronomy and Astrophysics 43: 381-383.

Thapa, R.R. and Hassan, M.R. 2013. Proceedings: Modern trends in science and Technology. Nepal Physical Society and Research Council of Science and Technology, Biratnagar, Nepal, 129-148p.

Vidyakin, V.V. 1994. Criterion of instability of translational rotational motion of perfectly rigid bodies, Soviet Astronomy 36: 214. 